\title{
Pour une nouvelle formation
}

Les nouveaux métiers de l'enseignement

New training for new professions in teaching

Para una nueva formación. Nuevos oficios en la enseñanza

\section{Goéry Delacôte}

\section{(2) OpenEdition}

12 Journals

Édition électronique

URL : http://journals.openedition.org/ries/2562

DOI : 10.4000/ries.2562

ISSN : 2261-4265

Éditeur

Centre international d'études pédagogiques

Édition imprimée

Date de publication : 1 mars 2000

Pagination : 35-39

ISSN : $1254-4590$

\section{Référence électronique}

Goéry Delacôte, «Pour une nouvelle formation », Revue internationale d'éducation de Sèvres [En ligne],

25 | 2000, mis en ligne le 01 mars 2003, consulté le 20 avril 2019. URL : http://

journals.openedition.org/ries/2562 ; DOI : 10.4000/ries.2562

\section{(c) Tous droits réservés}




\title{
Pour une nouvelle formation
}

\section{Les nouveaux métiers de l'enseignement}

\author{
Goéry Delacôte \\ Directeur général de l'Exploratorium de San Francisco
}

Je suis français, et heureux de l'être, et je dirige un musée des sciences en Californie, qui s'appelle l'Exploratorium. En même temps, j'ai travaillé avec l'Académie des sciences américaine à la promulgation des référents (standards) américains pour l'enseignement des sciences.

Vous savez peut-être que les Américains n'ont jamais défini leur enseignement sur le plan national. La Constitution américaine n'autorise pas que l'éducation soit une affaire nationale ou fédérale, c'est l'affaire des États ou des districts. Pour la première fois depuis deux siècles, les Américains se sont intéressés à la promulgation d'un cadre national de référence pour l'enseignement américain, c'est-à-dire for all Americans, pour tous les élèves. C'est une révolution tranquille, personne n'en a entendu parler. La raison est qu'elle est très peu controversée.

Nous avons pendant trois ans (de 1992 à 1995), avec l'Académie des sciences, avec des enseignants, des formateurs de maîtres, travaillé sur les contenus, la formation des maîtres, le contrôle des connaissances et toutes les méthodes d'évaluation. Nous avons réussi à promulguer, il y a deux ou trois ans, les standards in science education for all Americans, en quelque sorte les normes de ce que doit être l'enseignement des sciences pour tous aux États-Unis. De tels standards ont été promulgués en mathématiques, en sciences et dans d'autres disciplines. C'est donc la première fois, depuis la création des États-Unis, qu'il y a une réflexion politique nationale, sur un cadre de référence pour l'enseignement des différentes disciplines de la maternelle à l'université. Maintenant, cela se double d'une réflexion complémentaire qui vient de commencer il y a quelques jours à l'Académie pour les sciences de l'ingénieur de Washington (National Academy of Engineering). Il s'agit de travailler pendant deux ans avec des industriels, des enseignants, des universitaires, sur ce qu'il convient de faire pour augmenter la culture technologique de tous les Américains. Vaste sujet!

Aux États-Unis, il se passe beaucoup de choses en éducation, dont certaines représentent des évolutions très intéressantes - mais il y a aussi des régressions comme la résistance du Kansas à l'enseignement de la notion d'évolution en biologie. En particulier, il y a prise de conscience que les méthodes de transformation de l'enseignement américain, qui n'est pas en très bon état dans le primaire et dans le secondaire, pêchent essentiellement par une analyse trop peu stratégique, trop locale, trop fondée sur la méthode-projet, sur l'approche expérimentale et pas assez globale. Le président de l'Académie des sciences s'est fait le porte-parole de cette évolution pour l'enseignement des sciences. 
Les référents nationaux ne sont nullement obligatoires (savoir, enseignement, évaluation, formation des maitres...) contrairement à nos programmes, en France, qui se limitent à définir des contenus. Dans la pratique, certains États suivent les standards nationaux de très près, d'autres, d'un peu moins près, et d'autres encore, une minorité, pas du tout.

Dans mon établissement d'éducation informelle, l'Exploratorium, un musée des sciences, nous faisons de la formation de maitres venant de trente-deux États américains. Nous travaillons avec des formateurs de maitres en favorisant une approche fondée sur l'investigation et l'initiative de l'apprenant. C'est assez étonnant de voir qu'en fait, par des opérations de relativement courte durée, on peut arriver à engendrer cette conversion des enseignants, consistant à passer d'un mode traditionnel à un mode fondé sur l'investigation personnelle par l'élève. Il est plus difficile de maintenir cette conversion du maître, de garantir qu'il ne revienne pas aux pratiques antérieures. Pour éviter ce retour en arrière, il faut du temps avant, pendant, après la formation ; il faut les soutenir.

On va par ailleurs assister, dans les dix prochaines années, au départ en retraite d'une bonne moitié des enseignants, ce qui implique un important changement démographique. Les capacités actuelles de production de nouveaux enseignants sont sous-calibrées. Il est probable que ces changements démographiques seront à peu près les mêmes en Europe. C'est une chance, mais les gens ne prennent pas encore la mesure de cette chance pour mettre en place de nouvelles politiques de formation initiale ou de formation continuée.

Notre petite institution, l'Exploratorium, est devenue un centre national de formation des maîtres dans un esprit stratégique, très soutenu par la Fondation nationale des sciences (NSF), puisque l'on travaille avec la moitié des États américains. Plus généralement, l'Exploratorium est un centre de recherche et de développement d'outils d'apprentissage, et d'utilisation des multimédias dont certains peuvent servir à la formation des maitres en sciences. Ce n'est ni un musée, ni une université, ni une école, c'est un centre relais, un carrefour entre l'université, l'école, les familles, les gens des médias, le grand public, etc. Pour ce qui est des enseignants, nous avons maintenant une idée sur la manière de faire évoluer les maîtres, de les accompagner tout au long de leur carrière, depuis l'entrée dans le métier jusqu'à la sortie, mais avec d'importantes variations dans les modalités selon les époques.

Au début, il y a, bien entendu, la formation académique qui malheureusement aux États-Unis n'est pas toujours assurée, puisque la moitié des professeurs de mathématiques dans le secondaire n'ont jamais vraiment fait de mathématiques à l'université, même au niveau de la licence d'enseignement. C'est difficile de bien enseigner les mathématiques dans ces conditions, même à l'école primaire.

Ensuite, il y a une formation professionnelle qui dure une première année. Il y a beaucoup de choses à dire sur sa qualité, sur l'intérêt réel de la faire à l'école même, sur le terrain, dans des conditions de prises de décision éducative effective. Ce sujet est relativement bien connu. 
Ce qui est moins connu, et que nous avons réussi à faire comprendre à la Fondation nationale des sciences, qui finance maintenant de tels programmes, c'est la nécessité de soutenir les professeurs dans les cinq à sept premières années de leur activité professionnelle. On a constaté qu'aux États-Unis, le système éducatif perdait la moitié des enseignants de sciences dans les cinq premières années de leur carrière. Le système ne fonctionne donc pas bien.

Nous nous sommes proposé de trouver un mode d'accompagnement, dans les premières années de carrière, visant à ne pas garder tout le monde - car manifestement certains découvrent qu'ils ne sont pas faits pour cela - mais permettant de maintenir et de conforter une large proportion des enseignants qui, sans cet encadrement et ce soutien, auraient quitté l'éducation. La période des premières années est donc une phase d'induction, de consolidation, où l'enseignant fonctionne avec une sorte d'entourage, essentiellement sur son lieu de travail, parfois un peu endehors, qui fait largement appel bien sûr à des enseignants plus chevronnés, voire en situation de retraite (des enseignants qui viennent de partir).

Tout l'art est de gérer ce système, pour faire en sorte que l'enseignant ne soit plus isolé. L'expérience a si bien réussi que l'on nous a accordé un budget pour continuer à plus long terme.

Après, il y a le début de l'âge mûr, mais il faut continuer à se former. On voit apparaître un nouveau phénomène : la formation acquise passe beaucoup par l'acte de former - une sorte d'inversion. La meilleure formation acquise est celle qui consiste à donner une formation aux autres ou à aider les autres. On voit progressivement, dans les deux périodes suivantes de la carrière, que la formation des enseignants est optimale quand ceux-ci ont l'occasion de faire du développement, d'inverser leurs rôles (participation à des opérations de formation et de développement de nouveaux curricula...). Un exemple : celui de l'un des principaux centres de développement du curriculum, qui a travaillé dans tout le pays. À l'occasion d'un audit récent de ce centre (le Lawrence Hall of Science de l'université de Berkeley), nous avons d'abord regardé ensemble la carte d'installation des professeurs qui développaient leurs curricula, puis je leur ai demandé la carte des endroits où ces curricula étaient effectivement pratiqués par les enseignants. À ma grande surprise, j'ai découvert que la carte de diffusion du curriculum était à peine plus large que la carte des endroits d'expérimentation.

En réalité, tout cet effort de participation à la mise en place de curricula n'était qu'un extraordinaire outil de formation professionnelle. Je leur ai donc dit qu'au lieu de s'inquiéter du fait qu'il n'y avait pas beaucoup de diffusion au-delà du développement, il fallait au contraire considérer que le développement devenait un outil de formation professionnelle, et qu'il fallait simplement le gérer de manière à ce qu'il ne soit ni trop volumineux ni onéreux.

C'est certainement l'une des politiques qu'il faut arriver à mettre en place, c'est-à-dire conduire non pas à titre d'expérience, mais à titre de formation, des opérations de très large portée visant à mettre en place des outils nouveaux. Et cette inté- 
gration des enseignants dans la réflexion sur projet de ce qu'ils seront amenés à enseigner ou à transformer, est la meilleure formation professionnelle qu'on puisse leur donner, à condition de bien la calibrer, pour qu'elle ne soit pas trop lourde.

Enfin, dans la période de fin de carrière, il est certain que les enseignants auront acquis beaucoup de maturité. S'ils acceptent de donner la main à de jeunes collègues, sans être ni paternalistes ni encombrants, mais toujours sur des points précis comme l'évaluation, l'observation des élèves, l'utilisation des technologies, cela peut les maintenir dans une posture personnelle d'autoformation les amenant à terminer leur carrière en beauté, en restant utiles durant les premières années de leur retraite.

Il y a donc à repenser la fonction enseignante dans cette sorte de jeu subtil entre l'entrée, le milieu et la sortie de leur carrière. Il est banal de dire qu'il faut se former tout au long de la vie professionnelle, mais c'est un peu abstrait et peu différencié selon les périodes. Maintenant, en m'appuyant sur notre expérience de travail avec les enseignants, j'entrevois ces différentes phases et façons d'utiliser les compétences au cours des différents moments de la vie professionnelle.

Le dernier élément est que cette formation professionnelle, entre professeurs et sur le terrain, doit pour réussir avoir un certain nombre de composantes. On verra ainsi émerger des métiers nouveaux puisque le dispositif éducatif va changer en profondeur. On ne peut plus rester indéfiniment, tout le monde l'a dit, avec la formule d'un professeur, une classe, une heure et une discipline. Les technologies seront un très important levier de changement si des personnes intelligentes savent les utiliser stratégiquement.

Quelles sont les composantes à prendre en compte dans la formation ?

- mettre les enseignants dans une attitude de réflexion sur projet et de cocréation,

- savoirs fondamentaux : avoir un observatoire des idées clés, des attitudes, des concepts, rattachés,

- suivre en permanence l'évolution du champ conceptuel et des savoir-faire

- apprentissage : on sait maintenant beaucoup plus de choses sur l'apprentissage individuel,

- on a des idées sur ce qui peut rendre l'apprentissage efficace.

Actuellement, nous sommes dans une situation proche de celle où se trouvait la médecine avant l'arrivée de la biologie moléculaire. Le jour où l'on aura introduit ce savoir cognitif dans les pratiques d'apprentissage et d'enseignement comme nous le faisons chez nous, ou à Berkeley et à Stanford, on verra augmenter considérablement la qualité de l'enseignement et de l'apprentissage.

Quel sera l'impact de l'arrivée des nouvelles technologies ? Là aussi, la meilleure manière d'introduire les nouvelles technologies, ce n'est pas tant d'apprendre aux gens à s'en servir que de leur apprendre à réaliser de petites créations à partir de ces technologies (dessiner une page web, organiser un petit enseignement soutenu par des recherches effectuées sur le réseau...). 
Je travaille, par exemple, avec un groupe, le Concord Consortium dirigé par Robert Tinker, qui à Concord, près de Boston, développe très largement l'école virtuelle en marge de l'enseignement. Quand vous n'avez pas assez d'enseignants pour traiter d'un sujet, ou d'élèves dans une même école, il est intéressant de travailler avec l'école virtuelle puisqu'un enseignant travaille sur le thème pour des enfants de plusieurs écoles à la fois.

Toute cette dimension de l'organisation de l'apprentissage en réseau se fait pour le moment en marge du dispositif. Je crois qu'il ne faut pas s'y tromper, ces technologies arrivent et elles auront un rôle fondamental. Si le monde de l'éducation ne bouge pas, il est aujourd'hui entouré, demain il sera cerné. Et les principaux utilisateurs et consommateurs de l'éducation, les enfants, ont déjà accès à ce genre d'approche. D'où la nécessité d'avoir des endroits réels ou virtuels de création intelligente, de modes de conception d'espace virtuel, d'organisation de bases de données, capables de mettre en relation des personnes et d'accéder à des bases de savoirs utiles à des fins de résolution de problèmes. Il y a un énorme effort à faire, que les formateurs ne peuvent assurer tout seuls, mais qui doit être accompli dans un certain nombre d'établissements universitaires et dont les enseignants peuvent bénéficier.

L'Exploratorium a d'importants contrats pour la formation des enseignants parce que, justement, nous avons une expertise de tout premier plan, aux États-Unis, dans le développement de sites web ou de communication au travers du web (Webcasting) en relation avec les enseignants ou même conçus par eux. On peut sélectionner les meilleures démarches, les mettre en scène et les rendre interactives avec des centaines d'enseignants. On le réalise à l'endroit particulier où se trouve l'équipe qui a des caractéristiques définies. Cette méthode, plus inter-active que la télévision scolaire (temps réel, souplesse), offre un moyen intelligent d'utiliser les technologies au service d'une amélioration de l'apprentissage.

Donc, nouveaux savoirs, cognition humaine, utilisation des technologies, organisation du travail, sous des formes qui ne sont pas canoniques, sont les ingrédients fondamentaux des nouvelles méthodes d'apprentissage.

En résumé, il faut se former et former les autres pendant toute la carrière professionnelle; ne pas hésiter à cette occasion, à suivre l'évolution des savoirs, du développement de la cognition humaine et l'utilisation des technologies d'une manière intégrée. Pour réaliser ce projet, les enseignants ne peuvent pas être livrés à eux-mêmes, ils doivent être des opérateurs en termes de réflexion sur projet, aidés par des structures universitaires ou la création de nouveaux outils.

Il y a là une faiblesse, car on revient au problème du statut de la formation des enseignants dans les universités, qui est très généralement la dernière préoccupation des universitaires dont je suis membre. C'est un autre chantier très important. 